\title{
A Broadband Ultrathin Nonlinear Switching Metamaterial
}

\author{
Ehsan Zarnousheh Farahani ${ }^{1}$, Saughar Jarchi ${ }^{1}{ }^{*}$, Asghar Keshtkar ${ }^{1}$, \\ ${ }^{1}$ Department of Electrical Engineering, Faculty of Engineering and Technology, Imam Khomeini International University \\ (IKIU), Qazvin, Iran. \\ *corresponding author, E-mail: s.jarchi@eng.ikiu.ac.ir
}

\begin{abstract}
In this paper, an ultrathin planar nonlinear metamaterial slab is designed and simulated. Nonlinearity is provided through placing diodes in each metamaterial unit cell. The diodes are auto-biased and activated by an incident wave. The proposed structure represents a broadband switching property between two transmission and reflection states depending on the intensity of the incident wave. At low power states, around the second resonant mode of the structure's unit cell, the metamaterial slab presents high permittivity and therefore a near zero effective impedance, so the incident wave is reflected. Increasing the incident power to the level which can activate the loaded diodes in the structure results in elimination of the resonance and consequently a drop in the permittivity values near the permeability one as well as a switch to the transmission state. A full wave as well as a nonlinear simulations are performed. An optimization method based on weed colonization is applied to the unit cell of the metamaterial slab to achieve the maximum switching bandwidth. The structure represents a $24 \%$ switching bandwidth of a $10 \mathrm{~dB}$ reduction in the reflection coefficient.
\end{abstract}

\section{Introduction}

Many scientists have focused on this topic during the past decades by introducing metamaterial structures which have special electromagnetic characteristics not found in nature, for instance, negative $\mu_{\mathrm{r}}$ [1] and $\varepsilon_{\mathrm{r}}$ [2]. Scientists have demonstrated numerous applications of metamaterials such as cloaking [3], ultrathin absorber [4], antenna miniaturization [5], pattern modification [6], and reflector absorber structures [7]. The use of semiconductor devices with active biasing circuits has led to the expansion of these metamaterials into broader applications such as resonance frequency tuning [8] and beam steering [9]. Wave Transmission characteristics of metamaterial structures can also be altered using biased diodes[7,10]. However, active controlling circuits are not applicable to cases which need fast response to the intensity of the incident wave in microwave frequency ranges. These applications include high power limiting [11], switching applications [12], and harmonic generations [13]. Real-time controlling can be achieved by using nonlinearity of semiconductors and auto-biased passive controlling properties. Auto-biased control over semiconductors is provided by induced voltage across them as a result of the high intensity of the incident wave. Katko et al. [11] introduced a planar structure which decreases transmission coefficient by at least $3 \mathrm{~dB}$ in an $18 \%$ bandwidth by increasing the incident power to $30 \mathrm{dBm}$ level. Later Wall et al. [12] presented a bulk structure which decreases the reflection coefficient with an improved $30 \%$ in $3 \mathrm{~dB}$ bandwidth . This structure also showed an $18 \%$ bandwidth for an at least a $10 \mathrm{~dB}$ reduction in the reflection amplitude. Planar structures with a broad bandwidth have proven more useful in most practical applications [14].

In this paper, we designed and simulated a broadband planar switching metamaterial and provided the switching property through auto-biased diodes in metamaterial unit cells. A special feature of the proposed design is a relatively wide bandwidth provided by the planar structure. Thickness of the structure is $0.033 \lambda$, where $\lambda$ is wavelength of the center frequency, which is about 37 times thinner compared with [12]. In order to achieve the maximum switching bandwidth, we applied an optimization method based on weed colonization to the unit cell parameters and calculated the optimum values $[15,16]$. We applied the full wave as well as the nonlinear simulations to the metamaterial structure. Nonlinear simulations provide a confirmation for the structure performance $[17,18]$.

The paper is organized as follows. The theory of the structure and the proposed design are thoroughly discussed in section 2. The full wave simulations are provided in section 3 , where the optimization algorithm is applied to the structure and the optimum values for the maximum bandwidth are obtained. The nonlinear simulations are presented in section 4 , where nonlinearity of the diodes is also rigorously investigated. Finally, section 5 entails the concluding remarks.

\section{Theory and design}

\subsection{Theory}

According to the wave incident and reflection coefficients equations when an electromagnetic wave impinges onto a medium with different refractive indices and impedances, if impedance of the second medium, $\mathrm{Z}$, is purely imaginary, near zero or very high, the amplitude of the reflected wave will be near 1 , so the incident wave is reflected. Also by $\mathrm{Z}$ 
being near impedance of the first medium, $\mathrm{Z}_{0}$, there will be no reflection and all the incident wave will be transmitted. In order to have the desired reflection and transmission coefficients, the refractive index and intrinsic impedance of the medium must be engineered.

One of the main features of metamaterials is how much they can be engineered to show the desired effective intrinsic impedance in the microwave frequency band and therefore reflection or transmission characteristics.

Moreover, structures with different propagating states can be designed with applying nonlinearity in metamaterials. This nonlinearity can be achieved by using nonlinear devices such as diodes and transistors in each unit cell of the metamaterial structure. Different resistances and capacitances of a diode in its on and off states results in different metamaterial resonant behavior and thus different effective intrinsic impedances. In this paper, we have designed a structure which has two reflection and transmission states created by two on and off states of diodes, which are placed in the unit cell of the metamaterial. The diodes are auto-biased, i.e. no biasing circuit is needed in the structure. The reflection mode occurs because of the effective near zero intrinsic impedance of the structure and the off state of the diode. At high incident powers, the diodes are activated and the structure represents an effective intrinsic impedance near the free space impedance.

Electromagnetic characteristics of metamaterial structures such as effective permittivity and permeability and also intrinsic impedance and refraction index can be determined using the structures' $S$ parameters. In the numerical characterization, we simulated one unit cell with applying periodic boundary conditions on lateral directions. The structure is excited by a normally incident plane wave and $\mathrm{S}$ parameters are calculated. It is shown that [20]

$$
\begin{gathered}
S_{11}=\frac{R_{01}\left(1-e^{i 2 n k_{0} d}\right)}{1-R_{01}^{2} e^{i 2 n k_{0} d}} \\
S_{21}=\frac{\left(1-R_{01}^{2}\right) e^{i 2 n k_{0} d}}{1-R_{01}^{2} e^{i 2 n k_{0} d}}
\end{gathered}
$$

where $R_{01}$ is equal to $(z-1) /(z+1), n$ is refraction index, $k_{0}$ is the wave number, and $\mathrm{d}$ is the maximum slab length. Solving (1) and (2) gives [19] the following:

$$
\begin{gathered}
z= \pm \sqrt{\frac{\left(1+S_{11}\right)^{2}-S_{21}^{2}}{\left(1-S_{11}\right)^{2}-S_{21}^{2}}} \\
e^{i n k_{0} d}=\frac{S_{21}}{1-S_{11} \frac{z-1}{z+1}} \\
n=\frac{1}{k_{0} d}\left[\left\{\left[\ln \left(e^{i n k_{0} d}\right)\right]^{\prime \prime}+2 m \pi\right\}-\right. \\
\left.i\left[\ln \left(e^{i n k_{0} d}\right)\right]^{\prime}\right]
\end{gathered}
$$

where (.)' and (.)" respectively represent real and imaginary parts of the complex number and $\mathrm{m}$ is an integer representing the branch index of n'. These relations are valid as long as the unit cell dimensions are at most one fourth of the propagation wavelength so that the metamaterial slab can be considered as a homogenous medium[21]. There would be several solutions for the intrinsic impedance and refraction index. But we could calculate the correct solutions by considering the passive material conditions (real $(\mathrm{z}) \geq$ 0 and imaginary $(\mathrm{n}) \geq 0)$ [19]. Therefore $\epsilon=\mathrm{n} / \mathrm{z}$ and $\mu=\mathrm{nz}$ are calculated.

\subsection{Optimization Algorithm}

In order to improve the structure bandwidth optimization algorithms can be used. In $[15,16]$ an optimization algorithm inspired from weed colonization has been proposed and used for electromagnetic problems. To apply the method, firstly an initial population is created randomly. Then the Initial population is sorted based on the fitness function, which is higher bandwidth in this work. Depending on the fitness function, some individuals are selected as parents to produce seeds for next generation. The produced seeds are created with a Gaussian distribution function with standard deviation (SD), $\sigma$ and mean, $\mu$. In each step the standard derivation is calculated with the following equation:

$$
\sigma_{\text {iter }}=\frac{\left(\text { iter }_{\max }-\text { itrer }\right)^{n}}{\left(\text { iter }_{\max }\right)^{n}}\left(\sigma_{\text {initial }}-\sigma_{\text {final }}\right)+\sigma_{\text {final }}(6)
$$

The process will be repeated until maximum number of iterations. At last the individual with best fitness is chosen as the optimum solution.

\subsection{Metamaterial unit-cell}

In order to provide the desired reflection and transmission properties in the proposed metamaterial structure, we used electric elements in each unit cell, which affect the permittivity of the whole structure. Usually metamaterial structures which affect the permeability of the structure contain bulk unit cells to create loop currents in the structure. But electric metamaterials which modify the permittivity parameter could be designed in a planar and therefore ultrathin slab configuration. At frequencies near the resonance of the metamaterials consisting of electric unit cells such as cut wire $(\mathrm{CW})$, the electric split ring resonator (eSRR), thin wire (TW), and electric LC resonator (ELC), permittivity values have a high amplitude resulting in a near zero intrinsic impedance. The nonlinear characteristics could be achieved with diodes used in the unit-cells. At high incident powers impinging onto the metamaterial slab, the diodes are turned on and as a result the electric resonance is eliminated and the permittivity value drops to near the permeability one. This causes the slab to represent an impedance near the free space. The unit cell of the proposed structure and the TL-RLC model of it is illustrated in Fig. 1. A layer of FR4 with thickness of $1.6 \mathrm{~mm}$ is used as the substrate. On the top layer, six CWs of equal width and length are patterned in each unit cell. The bottom layer consists of eSRRs which are loaded with diodes. In Fig. 1 the front view is shifted up half a unit cell relative to the back view in $\mathrm{y}$ direction, because of better visualization of $\mathrm{CW}$ and SRR configurations used in front and back of the structure. The diodes are placed between adjacent unit cells. Two antiparallel schottky diodes are placed between two neighboring eSRRs. The incident wave has the linear polarization with electric field direction parallel with the CWs, each of the diodes is activated at half cycle of the induced electric field creating the activation voltage. The 
eSRR has two main electric resonances. The first one with a lower frequency can be attributed to an LC resonance created by the unit cell's loop inductance and the gap's capacitance (L1 and C1 shown in Fig. 1 (b) ) [22]. The second one, named the dipole resonant mode (L2 and C2 shown in Fig. 1 (b) ) [22], is caused by the sides which are parallel with the electric field of the induced wave. At frequencies near this resonance, the electric field is strong in the neighboring region where the diodes are placed. When the induced voltage across the diodes is more than the activation voltage, the diodes are activated and the resonance of the dipole mode is eliminated. We used the $\mathrm{CW}$ structure to tune the slab impedance at high power states. $\mathrm{CW}$ structure also act as an LC resonator which is shown with L3 and L4 in the structure's model presented in Fig. 1 (b). The effect of dielectric is modeled with the transmission line section.

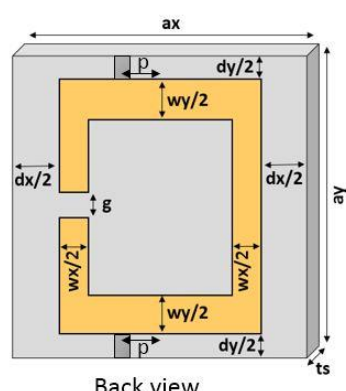

Back view

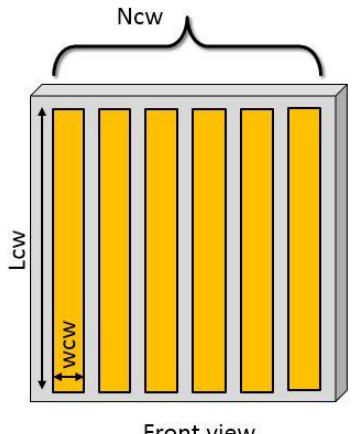

Front view (a)



(b)

Figure 1: Parametric view of unit cell (a). The front view is shifted up half a unit cell relative to the back view and parameter " $P$ " is the position of diodes relative to middle of the unit cell. TL-RLC model of eSRR-CW structure loaded with diode (b) [23].

\section{Full Wave Simulations}

We modeled the designed unit-cell in the CST microwave studio full wave simulation environment (Fig. 2) [24]. Diodes are modeled in their two on and off steady states. A
$2.5 \mathrm{k} \Omega$ resistance parallel with a $0.45 \mathrm{pF}$ capacitance is used to model the diodes off state and a $12 \Omega$ resistance to model the on state. These values result from the simulation of Skyworks SMS7621-075LF schottky diode spice model (based on the manufacturer's specifications) in the ADS environment [25], while inducing two $-10 \mathrm{dBm}$ and $30 \mathrm{dBm}$ power levels. FR4 is used for the substrate with $\epsilon_{r}=4.3$ and $\tan \delta=0.025$



(a)



(b)

Figure 2: Front (a) and back (b) side view of the unit cell's simulation model in CST studio environment, lumped element used as the diodes between two neighboring eSRRs.

In order to achieve the maximum operational bandwidth, the parameters of the unit cells are optimized. The optimization is achieved by the weed colonization method [16]. An initial population of 30 individuals was created randomly. The algorithm goal was achieved by a higher nonlinear operational bandwidth. Based on the first 15 individuals with 'best fit to the goal', the next generation was produced using a Gaussian distribution function [15]. After 15 generations, we reached an acceptable convergence. The individual with the best results was selected as the optimized structure.

The optimized parameters are illustrated in Table 1. The structure is simulated and S parameters are plotted in Fig. 3. In Full wave simulations, the incident power level is indicated by the lumped element models of the diodes in their on and off states. For low incident powers $(-10 \mathrm{dBm})$, the diodes are 
off and a totally reflective mode is observed in frequency band of $2.7-3.42 \mathrm{GHz}$. Permittivity and permeability of the structure are plotted in Fig. 4. The impedance of the structure for the low impedance powers is shown in Fig. 5 (a). High permittivity values and near zero impedance shown in these figures obviously represent the reason for the reflective behavior of the structure in low incident powers.

Table 1: Parameter values of designed unit cell.

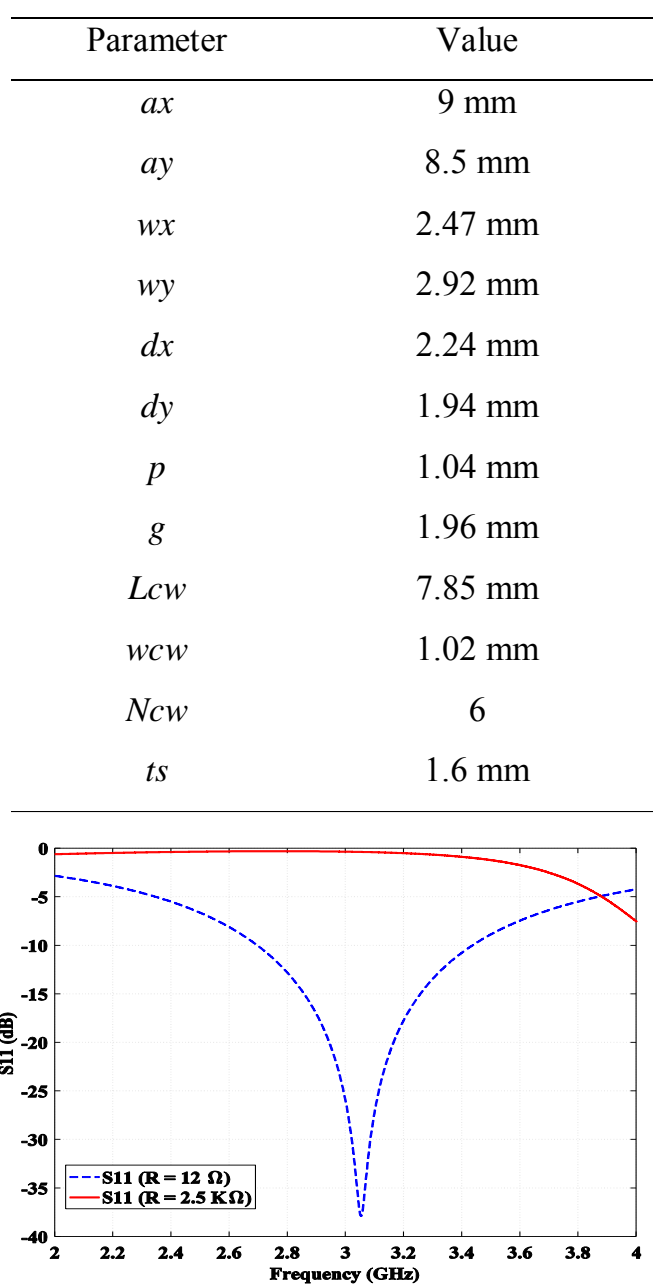

(a)

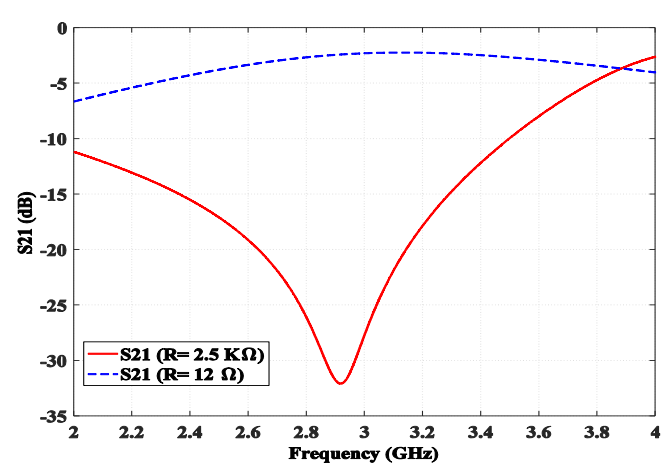

(b)

Figure 3: Simulated reflection (a) and transmission (b) characteristics of the structure for two states of the diodes.



(a)



(b)

Figure 4: Real parts of permittivity (a) and permeability (b) parameters at two reflection and transmission states.

At high incident power $(30 \mathrm{dBm})$, the diodes are activated and the structure behaves as a transmitter at the frequency band of $2-3.5 \mathrm{GHz}$. The permittivity and impedance of the structure are plotted in Figures 4 (a) and 5 (b), respectively. It is obvious in these figures that the permittivity values at this state drop near the permeability parameter and therefore the intrinsic impedance has values near the free space. A nonlinear switching bandwidth of $22.6 \%$, centered at 3.05 $\mathrm{GHz}$, with at least a $10 \mathrm{~dB}$ reduction in the reflection coefficient is achieved by the proposed structure. At this frequency band the maximum loss in the transmission mode is smaller than $2 \mathrm{~dB}$.

Transient simulation is also performed to show how the structure works in practice. The time domain response of the structure to an RF Gaussian pulse input signal including 2.7 $-3.4 \mathrm{GHz}$ is shown in Fig. 7 for the two reflection and transmission states. 




(a)

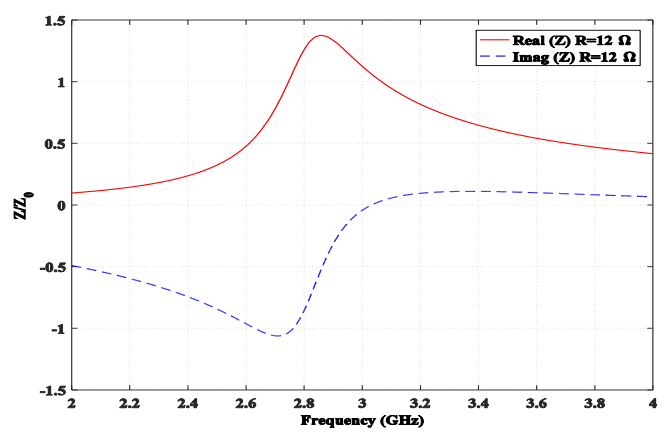

(b)

Figure 5: Real and imaginary parts of the structure's intrinsic impedance at two reflection (a) and transmission (b) states.

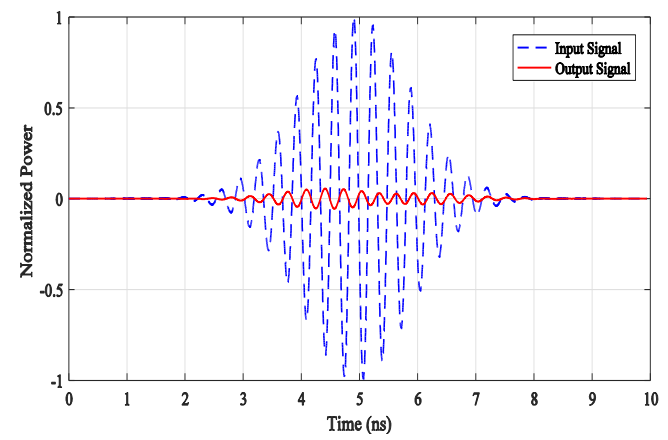

(a)

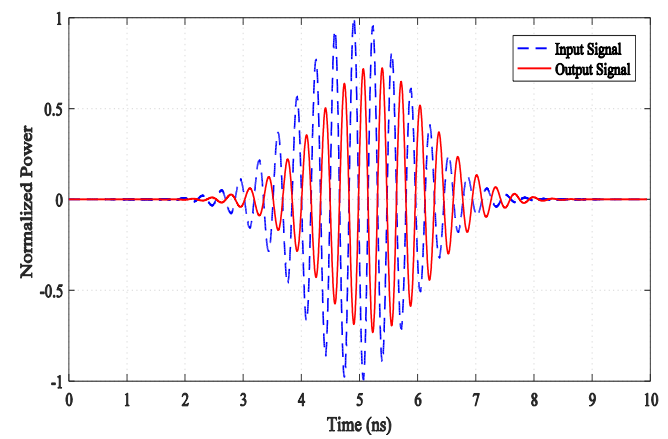

(b)

Figure 6: Time domain response of the structure to an RF Gaussian pulse input signal at two reflection (a) and transmission (b) states.
The y direction component of the electric field distribution on the eSRR unit cell side at the frequency of $3.05 \mathrm{GHz}$ is plotted in Fig. 7 (a). As illustrated in the figure, the magnitude of the electric field in the neighboring region of the unit cells where the diodes are placed is high. The induced voltage across the diodes can be calculated according to $\varphi=\int$ E. $\mathrm{dl}$ along the diodes. The voltage across the diodes for an incident power of 0.5 watts is calculated and plotted in Fig. 7 (b). As it can be seen in Fig. 7 (b) the voltage across the diodes all over the structure's working frequency band is higher than the activation voltage of the diodes $(260-320 \mathrm{mV}$, based on manufacturer's specifications); so the incident power would turn the diodes on.



(a)

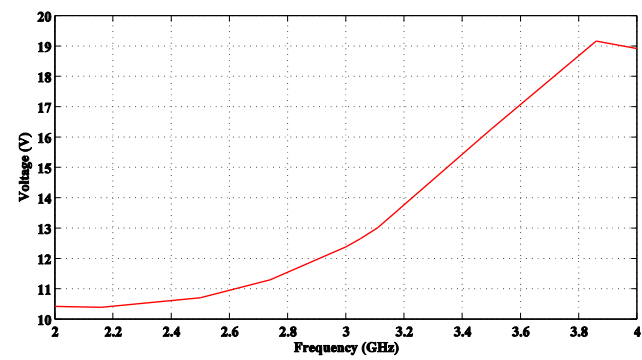

(b)

Figure 7: Magnitude of the electric field $\mathrm{E}_{\mathrm{y}}$ distribution on eSRR when the diodes are off (a) and induced voltage across diodes with an incident power intensity equal to 0.5 watts (b).

\section{Nonlinear Simulations}

Two main approaches for analyzing structures loaded with nonlinear elements are transient [18] and harmonic balance [17] simulations. We could carry them out by using the V-I relation model of the nonlinear element. In the harmonic balance method, the linear part of the structure is separated from the nonlinear element and then it is simulated by frequent full wave simulation softwares. Finally, the nonlinear solution is achieved by the V-I relation between the two linear and nonlinear parts in each harmonic simulation.

The full wave simulations showed the structure performance at two on and off steady states of the diodes using the lumped element models. To verify the dependence of the autobiasing behavior of the diodes on the high incident power, we 
applied a nonlinear analysis based on the harmonic balance method using the $\mathrm{S}$ parameters of the structure and nonlinear spice model of the diodes in the ADS environment. This was achieved by replacing the lumped element with the lumped port instead of the diode and then obtaining the $\mathrm{S}$ parameters of the structure with three ports in the full wave simulation environment. The $\mathrm{S}$ parameters obtained in the full wave simulation were imported into the ADS schematics environment and the third port was connected to the spice model of the diode, which could be used for nonlinear simulation. The other ports were connected to the single frequency power source ports and the large signal $\mathrm{S}$ parameters simulation block was used to run the nonlinear simulation, which uses the harmonic balance simulation method. The two $30 \mathrm{dBm}$ and $-10 \mathrm{dBm}$ wave power levels induced to the structure and $\mathrm{S}$ parameters are shown in Fig. 8. Comparing the nonlinear results to the full wave simulation results shows a quite good agreement in the switching performance of the structure but there is a little different in the resonance frequency. This is because of differences in the lumped element model of the diodes and the spice model.

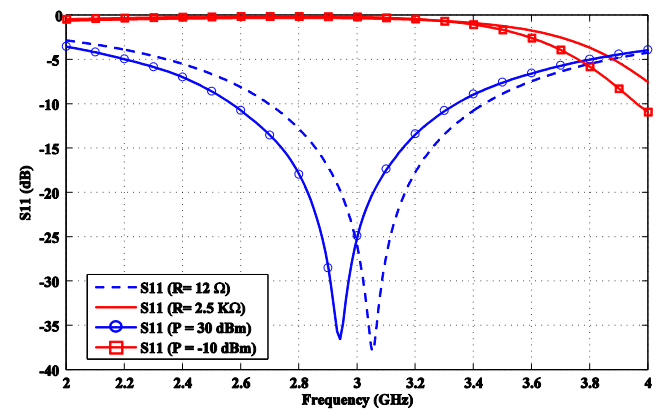

(a)

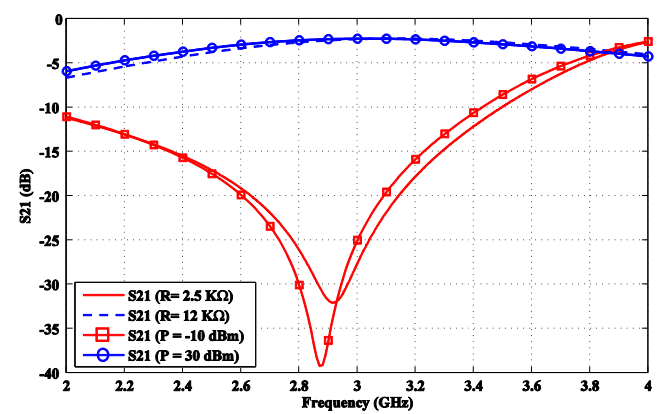

(b)

Figure 8: Comparison of S11 (a) and S21 (b) result from full wave and nonlinear simulations.

\section{Conclusions}

We designed and simulated a planar nonlinear switching metamaterial slab. The metamaterial unit cell was optimized to provide the maximum switching bandwidth. The full wave and nonlinear simulations verified the nonlinear broadband switching between the reflection and transmission behavior of the designed planar metamaterial structure. The unit cell of the structure consisted of the eSRR loaded with schottky diodes on one side and $\mathrm{CW}$ on the other side of the slab. The schottky diodes were placed to create nonlinear behavior and the CWs were used to tune the impedance of the structure on its transmission state. Nonlinearity is caused by activation of the diodes in response to the incident wave power. For high incident wave powers, the induced voltage across the diodes exceeds their activation voltage, which will turn on the diodes. Future works will focus on the experimental performance and measurement results.

\section{References}

[1] J. B. Pendry, A. J. Holden, D. J. Robbins, and W. J. Stewart, "Magnetism from conductors and enhanced nonlinear phenomena," Microwave Theory and Techniques, IEEE Transactions on, vol. 47, no. 11, pp. 2075-2084, 1999.

[2] J. B. Pendry, A. J. Holden, W. J. Stewart, and I. Youngs, "Extremely Low Frequency Plasmons in Metallic Mesostructures," Physical Review Letters, vol. 76, no. 25, pp. 4773-4776, 1996.

[3] J. B. Pendry, D. Schurig, and D. R. Smith, "Controlling Electromagnetic Fields," Science, vol. 312, no. 5781, pp. 1780-1782, 2006.

[4] N. I. Landy, S. Sajuyigbe, J. J. Mock, D. R. Smith, and W. J. Padilla, "Perfect Metamaterial Absorber," Physical Review Letters, vol. 100, no. 20, p. 207402, 2008.

[5] S. Jarchi, J. Rashed-Mohassel, and R. Faraji-Dana, "Proximity effects of a layered periodic structure on miniaturization of patch antennas," International Journal of RF and Microwave Computer-Aided Engineering, vol. 23, no. 5, pp. 549-558, 2013.

[6] A. Dadgarpour, B. Zarghooni, B. S. Virdee, and T. A. Denidni, "Beam Tilting Antenna Using Integrated Metamaterial Loading," Antennas and Propagation, IEEE Transactions on, vol. 62, no. 5, pp. 2874-2879, 2014.

[7] W. Xu and S. Sonkusale, "Microwave diode switchable metamaterial reflector/absorber," Applied Physics Letters, vol. 103, no. 3, p. 031902, 2013.

[8] Z. Jie, C. Qiang, C. Jie, Q. Mei Qing, J. Wei Xiang, and C. Tie Jun, "A tunable metamaterial absorber using varactor diodes," New Journal of Physics, vol. 15, no. 4, p. 043049, 2013.

[9] T. Jiang et al., "Low-DC Voltage-Controlled SteeringAntenna Radome Utilizing Tunable Active Metamaterial," Microwave Theory and Techniques, IEEE Transactions on, vol. 60, no. 1, pp. 170-178, 2012.

[10] B. Zhu, Y. Feng, J. Zhao, C. Huang, and T. Jiang, "Switchable metamaterial reflector/absorber for different polarized electromagnetic waves," Applied Physics Letters, vol. 97, no. 5, p. 051906, 2010.

[11] A. R. Katko, A. M. Hawkes, J. P. Barrett, and S. Cummer, "Rf limiter metamaterial using pin diodes," Antennas and Wireless Propagation Letters, IEEE, vol. 10, pp. 1571-1574, 2011.

[12] W. S. Wall, S. M. Rudolph, S. K. Hong, and K. L. 
Morgan, "Broadband switching nonlinear metamaterial," Antennas and Wireless Propagation Letters, IEEE, vol. 13, pp. 427-430, 2014.

[13] I. V. Shadrivov, A. B. Kozyrev, D. W. van der Weide, and Y. S. Kivshar, "Tunable transmission and harmonic generation in nonlinear metamaterials," Applied Physics Letters, vol. 93, no. 16, p. 161903, 2008.

[14] E. Zarnousheh Farahani, S. Jarchi, and A. Keshtkar, "Ultrathin Planar Nonlinear Switching Metamaterial," in 2nd International Conference on Electrical, Computer, Mechanical and Mechatronics Engineering, Istanbul, Turkey, 2015, p. 62: Science and Research Pioneers Institute.

[15] S. Jarchi, J. Rashed-Mohassel, M. Neshati, and C. Lucas, "A Dual Resonance Three Segment Rectangular Dielectric Resonator Antenna," in Progress In Electromagnetics Research Symposium Prague, Czech Republic, 2007, pp. 516-520: PIERS Proceedings.

[16] A. R. Mehrabian and C. Lucas, "A novel numerical optimization algorithm inspired from weed colonization," Ecological Informatics, vol. 1, no. 4, pp. 355-366, 2006.

[17] E. Zareian-Jahromi and J. Khalilpour, "Analysis of a Freestanding Frequency Selective Surface Loaded with a Nonlinear Element," Journal of Electromagnetic Waves and Applications, vol. 25, no. 2-3, pp. 247-255, 2011.

[18] S. M. Rudolph and W. S. Wall, "Nonlinear multiconductor transmission line analysis of broadband switching metamaterials," in Antennas and Propagation \& USNC/URSI National Radio Science Meeting, 2015 IEEE International Symposium on, 2015, pp. 75-76.

[19] D. M. Pozar, Microwave Engineering, 3rd ed. New York, NY, USA: Wiley, 2009.

[20] X. Chen, T. M. Grzegorczyk, B.-I. Wu, J. Pacheco, and J. A. Kong, "Robust method to retrieve the constitutive effective parameters of metamaterials," Physical Review $E$, vol. 70, no. 1, p. 016608, 2004.

[21] C. Caloz and T. Itoh, Electromagnetic metamaterials: transmission line theory and microwave applications. New York, NY, USA: Wiley, 2005.

[22] M. Kafesaki, K. Th, R. S. Penciu, T. F. Gundogdu, E. N. Economou, and C. M. Soukoulis, "Left-handed metamaterials: detailed numerical studies of the transmission properties," Journal of Optics A: Pure and Applied Optics, vol. 7, no. 2, p. S12, 2005.

[23] Q.-Y. Wen, Y.-S. Xie, H.-W. Zhang, Q.-H. Yang, Y.-X. Li, and Y.-L. Liu, "Transmission line model and fields analysis of metamaterial absorber in the terahertz band," Optics Express, vol. 17, no. 22, pp. 20256-20265, 2009.

[24] CST Studio Suite. French:Dassault Systèmes, 2015.

[25] Advanced Design System. United States:Keysight Technologies, 2016. 